\title{
To live is an act of courage...
}

\section{Sathiadas MG}

One of the main functions of medical schools is to train physicians to meet the healthcare needs of current and future national populations. This is usually achieved through arduous training curriculum and programmes in which high levels of motivation, intelligence and stamina are expected. This leads to immense stress levels and the extent of emotional disturbances amongst medical students is high. (1) Medical students have to cope with the complexity of the medical knowledge they learn, the financial debt that they sometimes have to take up, the lack of leisure time compared to their peers and having to make career choices at the end of medical school. The odds of preclinical students having been screened for depression was 1.63 times higher compared to clinical students. (1)

Suicide in doctors is common, and no other profession has a higher suicide rate (2). Schernhammer and Colditz conducted a meta-analysis of previous research on the issue and found that physicians had a higher suicide rate than the general population. Suicide risk related to burnout in doctors has been reported by several scholars. (3)

Burnout is a work-related syndrome consisting of emotional exhaustion, depersonalization, reduced personal accomplishment and having a negative feeling about oneself. This is first described nearly 30 years ago as a state of mental and/or physical exhaustion caused by excessive and prolonged stress. (4)

This stress state involving physiological and psychological responses can manifest as persistent irritability, persistent anxiety, periods of high blood pressure, insomnia, and forgetfulness. There may be palpitations, concentration problems, headaches and acute gastrointestinal symptoms. The deranged energy feeling may include some of the following symptoms: lateness for work, procrastination, decreased sexual desire, persistent tiredness in the mornings, social withdrawal (from friends and/or family), cynical attitudes, resentfulness, increased coffee/tea/cola and alcohol consumption, and ap- athy. The exhaustion stage may include chronic sadness or depression, chronic stomach or bowel problems, chronic mental fatigue, chronic physical fatigue, chronic headaches, the desire to "drop out" of society, the desire to move away from friends, work and even family, and perhaps the desire to commit suicide. (5)

Burned-out individuals may have a pessimistic view of the future and experience feeling of hopelessness and helplessness. A pessimistic view about the future as measured by the Beck Hopelessness Scale (BHS) has been reported as a major predictive factor for completed suicide even more so than depression or anxiety. (6)

Medical schools can be extremely stressful and lonely places. The teaching and learning environments can be extremely pressured, and expectations for achievement are high from the students themselves, their teachers, and parents. Three-quarters of psychiatric disorders in adulthood start during the early part of second decade and medical students are at the peak of training during this period hence high rates of psychiatric disorders in medical students manifest during this period.

A study done in Egypt by Okasha et al shows nearly $12.6 \%$ of medical students had reported some degree of suicidal feelings during the previous year. Around $5.6 \%$ of the participants had a maximum intensity of a feeling that life was not worthwhile whereas $4 \%$ had thought of taking their life, $0.9 \%$ had seriously considered suicide or made plans, and $0.4 \%$ had made an actual suicide attempt. (7) It is sad to note that the Jaffna Medical Faculty is faced with similar problem and urgent steps need to be taken to prevent further loss of precious lives.

Increasing awareness by a suicide awareness campaign and programme for early detection. Talking about it and alerting the students and families to watch for warning signs and letting them know that they are not alone. Medical schools must meet a minimum level of safety to be accredited. 
Minimising the stigma of mental health by addressing this in the curriculum and create a culture that it is not only ok but encouraged to seek mental health help as well. Threatening students, forcing medical leave for mental health issues can be detrimental and thereby shuts down communication. Students must be made aware that help is available and offer social/financial/academic resources. Establishing a suicide hotline or website messaging service which can answer anonymously to students in stressful situations. Screening all students annually for mental health issues by a medical examination can help in identifying the symptoms early.

Providing mentorship and peer support by creating a safe space in which students can talk freely and get help should be a regular activity in any student environment. Humanizing medical curriculum by giving opportunity to retake tests and learn clinically relevant material rather than molecular level facts is mandatory to reduce stress level. Helping medical students to be physically active would reduce depression and hopefully, suicide as it is known depression builds when we sit all day without doing anything. Learning environment must be free of bullying and stress from harsh teachers. Letting the students know it is okay to make mistakes and stumble and fall along the way will help them to learn coping mechanisms.

Self-management is vital and it involves the ability to learn how to relax and engage in scheduled exercise, rest and be able to pace oneself both inside and outside one's workplace. This will help to reorganize themselves, strengthen themselves and have better control over time and energy thereby reducing stress and getting suicidal ideation.

Lack of social support was two times the risk of suicide ideation among the University students when compared to students who had moderate or good social support. Suicide ideation was high among people who had less social support from their family, friends, and other relatives. (8) Not only the medical schools and working environment, the family, friends, and social network must be strong to prevent suicide among young adults.

There is an increase need for organisations to provide wellness programmes and regular workshops so that workers can participate. Research has shown that many physicians/workers do not participate in these programmes as it will reveal they are burnt out to their colleagues. (9) Such programmes should be part of the undergraduate curriculum so that students can feel it is normal to have symptoms of burnout. Normalisation of these issues and encouraging to seek help without inhibition will prevent undue stress among students as well as doctors.

\section{References}

1. Cuttilan, A. N., Sayampanathan, A. A., \& Ho, R. C. (2016). Mental health issues amongst medical students in Asia: a systematic review (2000-2015). Annals of translational medicine, 4(4), 72. https://doi.org/10.3978/j. issn.2305-5839.2016.02.07

2. Noonan D. Doctors who kill themselves. Newsweek 2008;151:16

3. Schernhammer ES, Colditz GA. Suicide rates among physicians: a quantitative and gender assessment (meta-analysis). Am J Psychiat 2004;161:2295-302

4. Pompili, Maurizio \& Innamorati, Marco \& Narciso, V \& Kotzalidis, Giorgio \& Dominici, Giovanni \& Talamo, A \& Girardi, Paolo \& Lester, David \& Tatarelli, Roberto. (2010). Burnout, hopelessness and suicide risk in medical doctors. La Clinica terapeutica. 161. 511-4.

5. Kuhn G, Goldberg R, Compton S. Tolerance for Uncertainty, Burnout, and Satisfaction With the Career of Emergency Medicine. Ann Emerg Med 2009

6. Beck AT, Weissman A, Lester D, et al. The measurement of pessimism: the hopelessness scale. J Consult Clin Psychol 1974; 42:861-5

7. Okasha A, Lotaif F, Sadek A. Prevalence of suicidal feelings in a sample of nonconsulting medical students. Acta Psychiatr Scand 1981;63:40915.

8. Desalegn, G.T., Wondie, M., Dereje, S. et al. Suicide ideation, attempt, and determinants among medical students Northwest Ethiopia: an institution-based cross-sectional study. Ann Gen Psychiatry 19, 44 (2020). https://doi. org/10.1186/s12991-020-00295-2

9. Medscape National Physician Burnout \& Suicide Report 2020: The Generational Divide 